\title{
Avaliação do desempenho dos diferentes métodos de interpoladores para parâmetros do balanço hídrico climatológico ${ }^{1}$
}

\author{
Fábio da S. Castro ${ }^{2}$, José E. M. Pezzopane ${ }^{2}$, Roberto A. Cecílioº ${ }^{2}$ José R. M. Pezzopane ${ }^{2}$ \& Alexandre C. Xavier ${ }^{2}$
}

\section{RESU MO}

A geoestatística está associada a uma classe de técnicas utilizadas para anal isar e inferir valores de uma variável distribuída no espaço ou no tempo, mediante o que se propôs, no presente trabalho avaliar, através de diferentes técnicas de interpolação, os seguintes parâmetros climáticos: precipitação, deficiência hídrica, excedente hídrico, evapotranspiração potencial, evapotranspiração real e disponibilidade hídrica, no estado do Espírito Santo. Para tanto, utilizaram-se dados meteorológicos de temperatura do ar e precipitação pluviométrica, compreendidos no período de 1977 a 2006, para 0 cálculo do balanço hídrico climatológico conforme método proposto por Thornthwaite \& Mather (1955), adotando uma capacidade de armazenamento de $100 \mathrm{~mm}$. Os resultados mostram que o método da krigagem é o mais eficiente para a espacialização dos parâmetros climáticos, baseado no menor valor da Raiz do Erro M édio Q uadrático (REM Q) e outros parâmetros calculados que auxiliaram na escolha do melhor modelo.

Palavras-chave: geoestatística, krigagem, interpolação

\section{Evaluation of the performance of the different methods of interpolaters for parameters of the climatologic water balance}

\begin{abstract}
Geostatistics is associated with a class of techniques used to analyze and to infer values of a variable distributed in space or time. By means of this, the objective of this work was to evaluate different techniques of interpolation for the following climatic parameters: precipitation, water deficit, water surplus, potential evapotranspiration, actual evapotranspiration and water availability in the State of the Espirito Santo. M eteorological data of air temperature and precipitation were used in the climatic water balance determination, according to Thornthwaite \& Mather (1955), adopting a storage capacity of $100 \mathrm{~mm}$. The results show that the method of kriging was the most efficient for the spatialization of climatic parameters, based on the lower value of the Root of the M ean Q uadratic Error (REM Q) and other calculated parameters that helped in choosing the best model.
\end{abstract}

Key words: geoestatistic, krigagem, interpolation

1 Parte da Dissertação de Mestrado do primeiro autor, apresentada à Universidade Federal do Espírito Santo

2 DEF/UFES - Alto Universitário, s/n, CP 16, CEP 29500-000, Alegre (ES). Fone: (28) 3558-1480. E-mail: fabiosilveira_70@hotmail.com; jemp@cca.ufes.br; rcecilio@cca.ufes.br, xavier@cca.ufes.br 


\section{INTRODUÇÃO}

A geoestatística vem apresentando aplicação crescente na avaliação da variabilidade espacial de parâmetros de interesse em ciências agrárias, permitindo o mapeamento, a quantificação e a modelagem de fenômenos contínuos, através da interpolação dos pontos amostrados no espaço (Vieira, 1997; Souza et al., 1998). Os interpoladores, por sua vez, são ferramentas matemáticas que atribuem valores relativos a alguma variável em pontos inseridos em um campo de valores já existente, transformando dados discretos em contínuos.

Tem-se utilizado em vários trabalhos, métodos de interpolação espacial para estimativas e espacialização de parâmetros climáticos; no entanto, muitos não têm atentado para a necessidade de se definir o melhor método de interpolação não existindo, até o momento, evidências de um método de interpolação que seja o melhor para diversas condições climáticas (Lennon \& Tunner, 1995). Até pouco tempo atrás, não se levava em consideração a preocupação com a qualidade da espacialização de dados climáticos feita pelos diferentes interpoladores, o que somente vem ocorrendo mais recentemente (Nalder \& Wein, 1998; Price et al., 2000; Taylor et al., 2004; Cecílio et al., 2006).

A qualidade de uma interpolação de dados depende da distribuição e do conhecimento dos pontos usados no cálculo, além da correlação de modelos estatísticos com os fenômenos em estudo. A escolha de um modelo apropriado é essencial para se obter resultados aceitáveis (Aranoff, 1989). Mediante este fato é importante avaliar o desempenho dos interpoladores para cada variável estudada.

Os elementos do tempo e clima afetam diretamente o crescimento e o desenvolvimento das plantas sobre diferentes formas e nas diversas fases do ciclo da cultura; em assim sendo, o conhecimento da variabilidade espacial dos elementos climatológicos, como deficiência hídrica do solo, excedente hídrico, evapotranspiração potencial, evapotranspiração real, armazenamento de água no solo e a disponibilidade hídrica (ER/ETP), se torna indispensável no planejamento e implantação de uma atividade agrícola (Pereira et al., 2002; Black, 2007; Sentelhas et al., 2008).

Do exposto, o presente trabalho tem por objetivo avaliar o desempenho dos métodos de interpoladores Inverso de uma potência da distância (IPD) e krigagem (geoestatístico) para espacialização das variáveis climatológicas: precipitação, excedente hídrico, deficiência hídrica, evapotranspiração potencial, evapotranspiração real e disponibilidade hídrica, no estado do Espírito Santo.

\section{MATERIAL E MÉTODOS}

\section{Localização da área de estudo}

A área contemplada no presente estudo é o estado do Espírito Santo, com área total de $46.184,1 \mathrm{~km}^{2}$, situado geograficamente entre os meridianos $39^{\circ} 38^{\prime}$ e $41^{\circ} 50^{\prime}$ de longitude Oeste e os paralelos $17^{\circ} 52^{\prime}$ e $21^{\circ} 19^{\prime}$ de latitude Sul.

\section{Dados meteorológicos utilizados no estudo}

Séries históricas de temperaturas médias do ar e total mensal de precipitação pluvial foram utilizadas em escala mensal e anual, considerando-se um período de 30 anos de dados (1977-2006).

Obtiveram-se, para o estado do Espírito Santo, dados meteorológicos de 94 postos de medição, dos quais 11 pertencentes à rede de estações meteorológicas do Instituto Capixaba de Pesquisas e Extensão Rural (INCAPER), 3 ao Instituto Nacional de Meteorologia (INMET), que medem temperatura do ar e precipitação; e outros 80 pertencentes à Agência Nacional de Águas (ANA), que medem somente a precipitação. Adotaram-se, ainda, outros 16 postos pluviométricos pertencentes também à ANA, localizados fora do estado, cujo objetivo foi minimizar o efeito de borda no processo de interpolação. No total, foram utilizados 110 pontos de medição cuja distribuição espacial é apresentada na Figura 1.

Os registros, que pertenciam aos postos pluviométricos da ANA, foram adquiridos gratuitamente através do sistema de informações hidrológicas (Hidroweb) disponibilizado no endereço eletrônico http://hidroweb.ana.gov.br.

As informações meteorológicas referentes às estações do INCAPER e do INMET estavam sob a responsabilidade da equipe do INCAPER/SIAG (Sistema de Informações Agro-

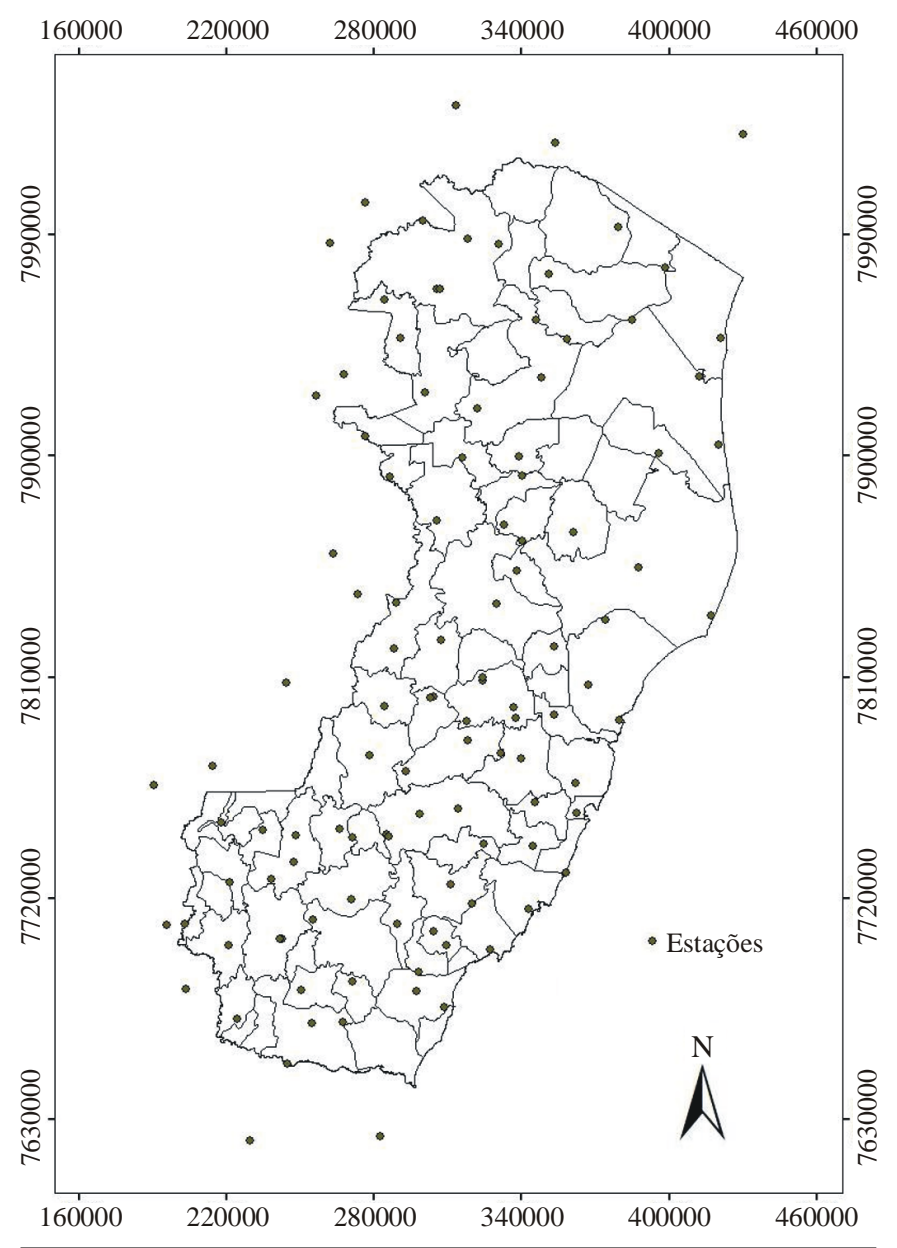

Figura 1. Distribuição espacial dos postos de medição utilizados no presente estudo 
meteorológicas), e foram adquiridas através de visitas ao órgão e consulta ao seu banco de dados.

\section{Preenchimento das falhas de precipitação}

Os dados referentes a alguns dos postos de medição pertencentes à ANA apresentavam falhas nos registros de precipitação pluvial nas séries mensais, as quais foram preenchidas utilizando-se o método da ponderação regional, devido á sua simplicidade e maior aplicabilidade para o preenchimento de séries mensais ou anuais de precipitações pluviométricas (Bertoni \& Tucci, 2002). Para a aplicação do método foram selecionados os três mais próximos daquele que apresentava falhas e se estimou o valor da precipitação por meio da Eq. 1.

$$
\mathrm{Yc}=\frac{1}{3}\left[\frac{\mathrm{X}_{1}}{\mathrm{Xm}_{1}}+\frac{\mathrm{X}_{2}}{\mathrm{Xm}_{2}}+\frac{\mathrm{X}_{3}}{\mathrm{Xm}_{3}}\right] * \mathrm{Ym}
$$

em que:

$$
\begin{aligned}
& \text { Yc - precipitação do posto a ser estimada } \\
& \mathrm{X}_{1}, \mathrm{X}_{2} \text { e } \mathrm{X}_{3} \text { - precipitações correspondentes ao mês }
\end{aligned}
$$

Esta etapa foi fundamental para a homogeneização do período de informações e análise estatística da precipitação na base de dados garantindo, assim, maior confiabilidade e exatidão das informações na elaboração do trabalho.

\section{Balanço hídrico climatológico}

Mediante os dados de temperatura média do ar e precipitação pluviométrica para todas as localidades, calculou-se o balanço hídrico climático mensal, pelo método proposto por Thornthwaite \& Mather (1955), com o auxílio do programa "BHnorm", elaborado em planilha por Rolim et al. (1998), assumindo-se uma Capacidade Máxima de Armazenamento de água no solo (CAD) igual a $100 \mathrm{~mm}$.

A evapotranspiração potencial foi estimada pelo método de Thornthwaite (1948); trata-se de um método empírico baseado apenas na temperatura média do ar e nas coordenadas geográficas do local. Escolheu-se este método pela disponibilidade dos dados climáticos e por ter sido desenvolvido para regiões de clima úmido, apresentando boas estimativas para as condições do estado (Pereira et al., 2002).

Os parâmetros do balanço hídrico calculados, foram evapotranspiração real (ER), armazenamento de água no solo (ARM), negativo acumulado (NEG ACUM) alteração de água no solo (ALT), deficiência hídrica no solo (DEF) e excedente hídrico $(\mathrm{EXC})$.

\section{Avaliação do desempenho dos métodos de interpoladores}

Para se proceder ao estudo da variabilidade e da dependência espacial dos valores de determinado atributo, é necessário que cada amostra seja associada à sua respectiva posição relativa ou coordenada espacial.
Os parâmetros avaliados para a realização da espacialização, foram precipitação $(\mathrm{P})$, deficiência hídrica (DEF), evapotranspiração potencial (ETP), evapotranspiração real (ER), excedente hídrico (EXC) e disponibilidade hídrica (ER/ETP).

Realizou-se a análise espacial da dependência dos dados por intermédio de um programa desenvolvido no software Matlab 2007 (MathWorks, 2007), que utiliza os valores da variável em estudo com suas respectivas coordenadas de campo para a construção do semivariograma experimental (Xavier et al., 2010).

Os resultados dos valores gerados pelo balanço hídrico passaram por uma análise descritiva a fim de se verificar alguma anormalidade dos mesmos, realizando-se, logo em seguida, uma análise geoestatística visando quantificar o grau de dependência espacial dos dados, através de um semivariograma experimental, estimado pela Eq. 2 .

$$
\gamma^{*}(\mathrm{~h})=\frac{1}{2 \mathrm{~N}(\mathrm{~h})} \sum_{\mathrm{i}=1}^{\mathrm{N}(\mathrm{h})}\left[\mathrm{Z}\left(\mathrm{x}_{\mathrm{i}}\right)-\mathrm{Z}\left(\mathrm{x}_{\mathrm{i}}+\mathrm{h}\right)\right]^{2}
$$

em que:

$$
\begin{aligned}
& \gamma^{*}(\mathrm{~h})- \text { valor do semivariograma estimado para a } \\
& \text { distância } \mathrm{h} \\
& \mathrm{N}(\mathrm{h})- \text { número de pares de valores medidos } \\
& \mathrm{x}_{\mathrm{i}} \text { e } \mathrm{x}_{\mathrm{i}}+\mathrm{h}-\begin{array}{l}
\text { locais de amostragens separados por uma } \\
\text { distância } \mathrm{h}
\end{array}
\end{aligned}
$$

$\mathrm{Z}\left(\mathrm{x}_{\mathrm{i}}\right)$ e $\mathrm{Z}\left(\mathrm{x}_{\mathrm{i}}+\mathrm{h}\right)$ - valores medidos das variáveis nos locais correspondentes

O software fez o ajuste automático dos modelos teóricos - exponencial, esférico, gaussiano e linear, com patamar ao semivariograma experimental, para o método da krigagem, de modo que a curva que melhor se ajustar aos pontos obtidos represente a magnitude, alcance e intensidade da variabilidade espacial da variável estudada. Avaliou-se, também, o desempenho para o método do inverso de uma potência da distância (IPD) para os níveis de potência de 1 a 5 .

\section{Validação cruzada dos dados e escolha dos modelos}

Diante dos modelos de semivariograma experimental, realizou-se a validação cruzada dos dados de todos os interpoladores, através da metodologia apresentada em Robinson \& Metternicht (2006) e Amorim et al. (2008) na qual um posto específico é descartado sucessivamente na realização da interpolação; assim, é possível obter o valor estimado (E) relativo ao posto retirado e, posteriormente, compará-lo com o valor real da variável $(\mathrm{O})$, porém todo este processo descrito acima foi automatizado garantindo, desta forma, maior agilidade nas operações.

Através dos índices estatísticos fornecidos pela validação cruzada dos dados foi possível realizar a avaliação do desempenho dos interpoladores e seleção do modelo utilizando-se, como um dos critérios de decisão, o menor valor da raiz do erro médio quadrático (REMQ), conforme Legates \& Mccabe Jr. (1999) definido pela Eq. 3.

$$
\text { REQM }=\sqrt{\frac{\sum_{i=1}^{J}\left(O_{i}-E_{i}\right)^{2}}{J}}
$$


em que:

$$
\begin{aligned}
& \text { J - número de observações } \\
& \mathrm{O} \text { - valor observado experimentalmente } \\
& \mathrm{E} \text { - valor estimado pelo método }
\end{aligned}
$$

Camargo \& Sentelhas (1997) propõem que, ao se correlacionar os valores estimados e os observados experimentalmente, sejam considerados o coeficiente de correlação (r) e o índice de concordância (d). Ainda segundo os autores, a precisão do modelo é dada pelo coeficiente de correlação (r) e a exatidão está relacionada ao afastamento dos valores estimados em relação aos observados; matematicamente, esta aproximação é dada pelo índice de concordância (d), seus valores variam de zero para nenhuma concordância a 1 para a concordância perfeita. $\mathrm{O}$ valor de (d) foi definido através da Eq. 4.

$$
d=1-\frac{\sum_{i=1}^{J}\left(O_{i}-E_{i}\right)^{2}}{\sum_{i=1}^{J}\left(\left|O_{i}-\bar{O}\right|+\left|O_{i}-\bar{O}\right|\right)^{2}}
$$

em que $\overline{\mathrm{O}}$ é a média dos valores observados experimentalmente.

O índice de confiança (c) proposto por Camargo \& Sentelhas (1997) permite analisar conjuntamente a precisão e a exatidão dos resultados obtidos, através do produto do coeficiente de correlação (r) pelo índice de concordância (d).

Os critérios de avaliação do desempenho de modelos quanto ao índice de confiança, estão apresentados na Tabela 1.

Tabela 1. Análise do desempenho do modelo com base no índice de confiança

\begin{tabular}{cc}
\hline Valor de c & Desempenho \\
$>0,85$ & Ótimo \\
0,76 a 0,85 & Muito bom \\
0,66 a 0,75 & Bom \\
0,61 a 0,65 & Mediano \\
0,51 a 0,60 & Sofrível \\
0,41 a 0,50 & Mau \\
$\leq 0,40$ & Péssimo \\
\hline
\end{tabular}

O coeficiente de eficiência ajustado ( $E$ ') também é um índice importante e vem sendo utilizado para a avaliação de vários modelos hidrológicos (Wilcox et al., 1990; Yu, 1999). Legates \& Mccabe Jr. (1999) definiram este índice, que varia de $-\infty$ a 1 , com os maiores valores indicando melhor performance, calculado por intermédio da Eq. 5.

$$
E^{\prime}=1-\frac{\sum_{i=1}^{J}\left|O_{i}-E_{i}\right|}{\sum_{i=1}^{J}\left|O_{i}-\bar{O}\right|}
$$

Além disso, os mesmos autores indicam que, como informação adicional, deve ser incluído o erro médio absoluto (EMA), definido pela Eq. 6.

$$
\text { EMA }=\frac{\sum_{i=1}^{J}\left|O_{i}-E_{i}\right|}{J}
$$

Finalmente, Chong et al. (1982) utilizam o erro médio percentual (EMP), expresso pela Eq. 7, porém a referida equação tende a apresentar valores altíssimos de EMP, quando os valores observados $\left(\mathrm{O}_{\mathrm{i}}\right)$ são baixos.

$$
\mathrm{EMP}=\frac{\sum_{\mathrm{i}=1}^{\mathrm{J}} \frac{\left|\mathrm{O}_{\mathrm{i}}-\mathrm{E}_{\mathrm{i}}\right|}{\mathrm{O}_{\mathrm{i}}}}{\mathrm{J}} * 100
$$

Visto que eram muitos os valores reais 0 (zero) nos dados de algumas variáveis, e se sabe que este é um limite físico que não é atendido pelo critério matemático da interpolação, adotaram-se valores reais muito próximos a zero $(0,001)$ para substituí-los nos resultados do balanço hídrico e, posteriormente, realizar a geração dos semivariogramas e avaliação do desempenho dos interpoladores.

\section{RESULTADOS E DISCUSSÃO}

Com base na validação cruzada dos dados, as Tabelas 2 a 7 apresentam os índices estatísticos calculados que auxiliaram na escolha do melhor método e modelo de interpolação para as variáveis climáticas estudadas.

A Tabela 2 apresenta os índices estatísticos calculados para analisar o desempenho dos interpoladores para a espacialização da precipitação média anual, no estado do Espírito Santo.

De acordo com o critério definido por Camargo \& Sentelhas (1997), o modelo krigagem exponencial foi considerado "bom" para a estimativa dos valores de precipitação para confecção do mapa temático.

$\mathrm{O}$ método krigagem exponencial demonstrou-se mais eficiente para estimativa das precipitações médias anuais no estado do Espírito Santo, pois apresenta uma correlação satisfatória entre os valores reais e os valores estimados, baseado no menor REMQ e nos outros índices estatísticos que apontam nesta direção.

Os dados da Tabela 2 mostram variação de EMP entre 7,72 e 9,54\%; em média o erro percentual do melhor interpolador é muito baixo, em se tratando de dados de precipi-

Tabela 2. Índices estatísticos calculados visando à análise do desempenho dos interpoladores para estimativa da precipitação média anual, no Espírito Santo

\begin{tabular}{lcccccccc}
\hline Modelo & $\mathbf{R}^{2}$ & REMQ & $\mathbf{d}$ & $\mathbf{c}$ & Desempenho & E' & EMA & $\begin{array}{c}\text { EMP } \\
\text { (\%) }\end{array}$ \\
Exponencial & 0,59 & 124,84 & 0,86 & 0,66 & Bom & 0,40 & 95,31 & 7,72 \\
Esférico & 0,58 & 126,42 & 0,86 & 0,65 & Mediano & 0,39 & 97,04 & 7,91 \\
Gaussiano & 0,43 & 158,67 & 0,81 & 0,53 & Sofrível & 0,23 & 119,33 & 9,54 \\
Linear & 0,57 & 128,18 & 0,86 & 0,65 & Mediano & 0,39 & 97,16 & 7,79 \\
IPD 1 & 0,43 & 148,31 & 0,78 & 0,51 & Sofrível & 0,28 & 115,03 & 9,40 \\
IPD 2 & 0,44 & 147,50 & 0,79 & 0,53 & Sofrível & 0,29 & 113,21 & 9,24 \\
IPD 3 & 0,44 & 148,63 & 0,79 & 0,53 & Sofrível & 0,30 & 111,82 & 9,13 \\
IPD 4 & 0,43 & 150,92 & 0,80 & 0,52 & Sofrível & 0,30 & 112,25 & 9,15 \\
IPD 5 & 0,42 & 153,51 & 0,79 & 0,52 & Sofrível & 0,29 & 113,68 & 9,27 \\
IPD 6 & 0,41 & 155,97 & 0,79 & 0,51 & Sofrível & 0,28 & 115,42 & 9,41 \\
\hline
\end{tabular}

$\mathrm{R}^{2}$ - coeficiente de determinação da validação cruzada; REMQ - raiz do erro médio quadrático; d - índice de concordância; $c$ - índice de confiança; $E^{\prime}$ - coeficiente de eficiência ajustado; EMA - erro médio absoluto; e EMP (\%) - erro médio percentual 
tação que, sabidamente, apresentam grande variação espacial, sobretudo no Espírito Santo, onde ocorre grande influência orográfica na precipitação.

Embora ainda se utilizem em alguns trabalhos, do método IPD2 para espacialização da precipitação média anual (Barbosa et al., 2005; Amorim et al., 2008), parece haver um consenso entre pesquisadores, de que o método Krigagem apresenta melhor desempenho para interpolação deste elemento do clima em diversas regiões do País, conforme mostram os trabalhos de Carvalho \& Assad (2005), Barbosa
(2006) e Silva et al. (2007), corroborando, desta forma, com os resultados encontrados no presente estudo. A Figura 2 apresenta os mapas de distribuição espacial da precipitação no Espírito Santo utilizando-se o método da krigagem exponencial (Figura 2A) e IPD2 (Figura 2B). Nota-se sensível diferença entre os mapas, sobremaneira nas proximidades dos postos pluviométricos que registraram os valores extremos (mínimos e máximos) de lâmina precipitada, havendo, no mapa gerado por IPD2, maiores áreas com valores próximos aos extremos de altura pluviométrica anual.
A.

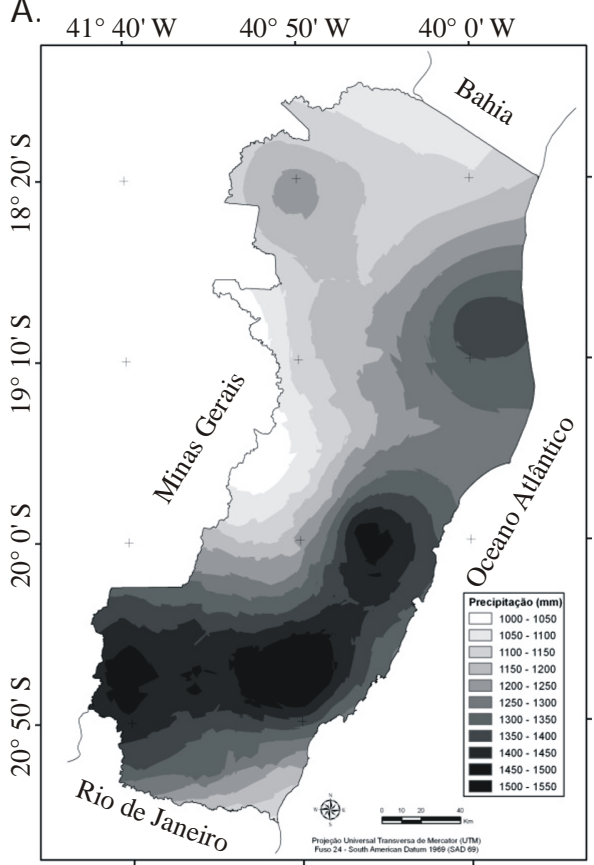

D. $41^{\circ} 40^{\prime} \mathrm{W}$ $40^{\circ} 50^{\prime} \mathrm{W}$ $40^{\circ} 0^{\prime} \mathrm{W}$

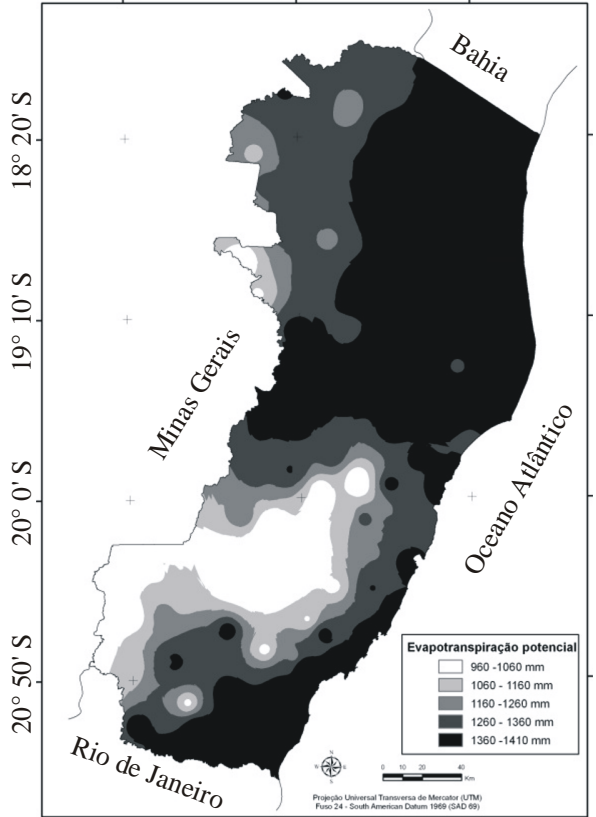

$41^{\circ} 40^{\prime} \mathrm{W} \quad 40^{\circ} 50^{\prime} \mathrm{W} \quad 40^{\circ} 0^{\prime} \mathrm{W}$
B.

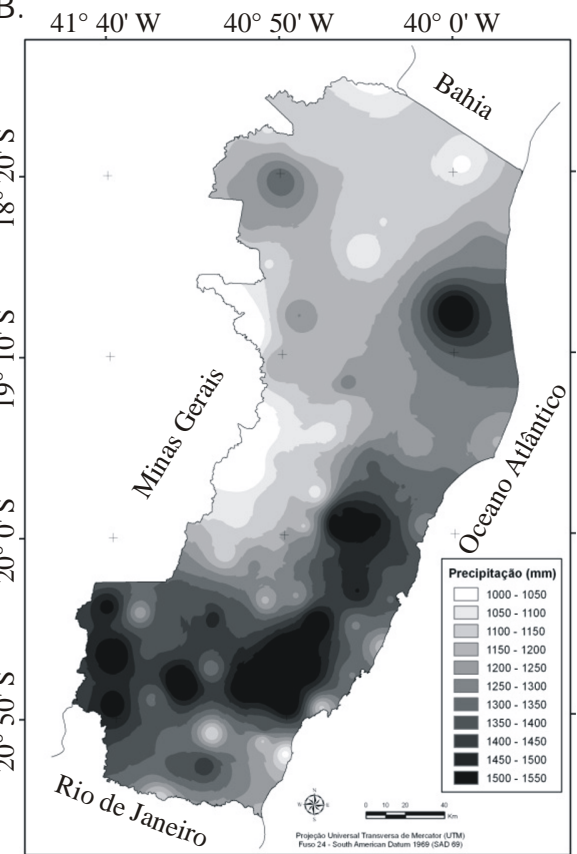

E. $41^{\circ} 40^{\prime} \mathrm{W}$

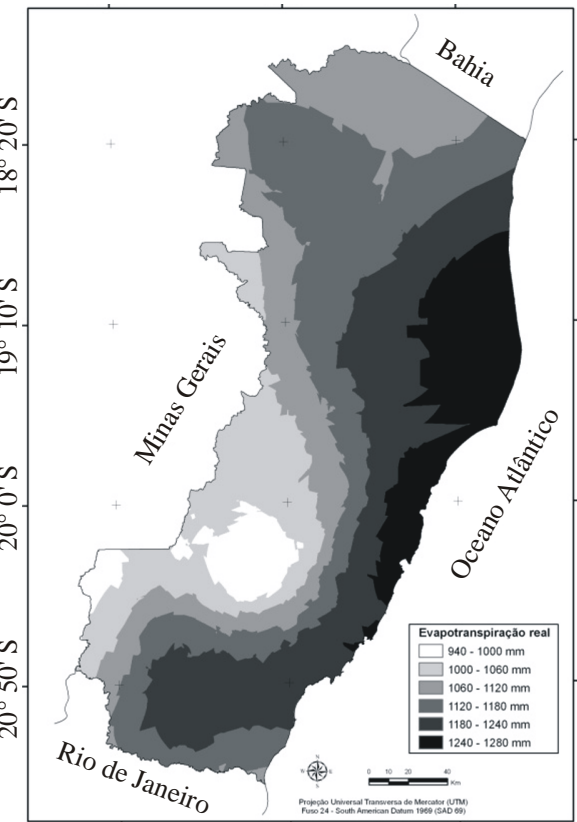

$41^{\circ} 40^{\prime} \mathrm{W} \quad 40^{\circ} 50^{\prime} \mathrm{W}$
C.

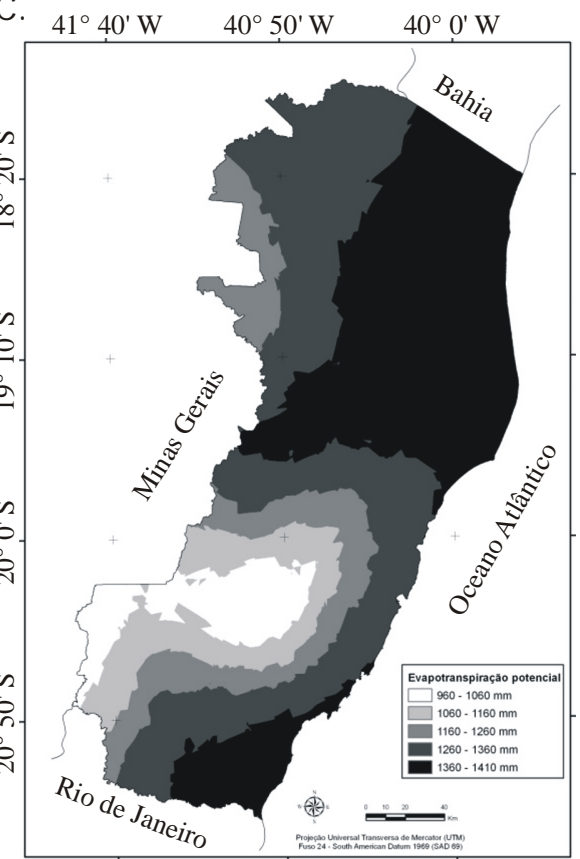

F. $41^{\circ} 40^{\prime} \mathrm{W} \quad 40^{\circ} 50^{\prime} \mathrm{W} \quad 40^{\circ} 0^{\prime} \mathrm{W}$

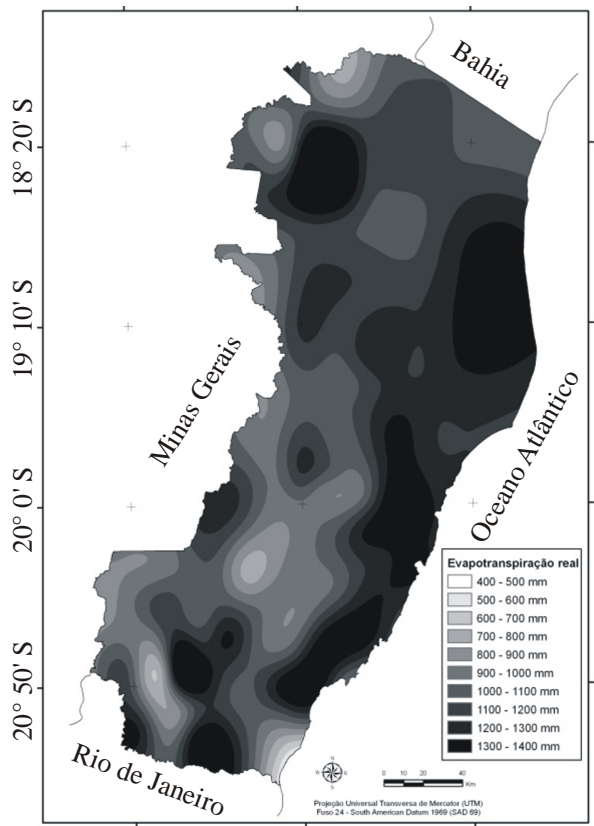

$41^{\circ} 40^{\prime} \mathrm{W} \quad 40^{\circ} 50^{\prime} \mathrm{W} \quad 40^{\circ} 0^{\prime} \mathrm{W}$

Figura 2. Distribuições espaciais da precipitação anual no Espírito Santo, obtidas por meio de krigagem exponencial (A); inverso do quadrado da distância (B); da evapotranspiração potencial anual no Espírito Santo, obtidas por meio de krigagem esférica (C); inverso do quadrado da distância (D); da evapotranspiração real anual no Espírito Santo, obtidas por meio de krigagem exponencial (E) e spline (F) 
A Tabela 3 apresenta os índices estatísticos calculados para analisar o desempenho dos interpoladores para a espacialização da evapotranspiração potencial média anual no estado do Espírito Santo.

Tabela 3. Índices estatísticos calculados visando à análise do desempenho dos interpoladores para estimativa da evapotranspiração potencial média anual, no estado do Espírito Santo

\begin{tabular}{|c|c|c|c|c|c|c|c|c|}
\hline Modelo & $\mathbf{R}^{2}$ & REMQ & d & c & Desempenho & $\mathbf{E}^{\prime}$ & EMA & $\begin{array}{r}\text { EMP } \\
(\%)\end{array}$ \\
\hline Exponencial & 0,59 & 115,48 & 0,86 & 0,66 & Bom & 0,44 & 85,61 & 7,15 \\
\hline Esférico & 0,58 & 114,74 & 0,87 & 0,66 & Bom & 0,44 & 84,60 & 7,09 \\
\hline Linear & 0,45 & 159,35 & 0,82 & 0,55 & Sofrível & 0,29 & 109,88 & 8,66 \\
\hline Gaussiano & 0,58 & 116,50 & 0,86 & 0,66 & Bom & 0,43 & 86,66 & 7,22 \\
\hline IPD 1 & 0,49 & 127,71 & 0,80 & 0,56 & Sofrível & 0,37 & 96,88 & 8,20 \\
\hline IPD 2 & 0,49 & 127,81 & 0,81 & 0,57 & Sofrível & 0,37 & 95,97 & 8,11 \\
\hline IPD 3 & 0,47 & 131,01 & 0,81 & 0,56 & Sofrível & 0,36 & 98,25 & 8,31 \\
\hline IPD 4 & 0,45 & 135,32 & 0,81 & 0,54 & Sofrível & 0,34 & 101,39 & 8,57 \\
\hline IPD 5 & 0,43 & 139,41 & 0,80 & 0,52 & Sofrível & 0,32 & 104,24 & 8,80 \\
\hline IPD 6 & 0,41 & 142,85 & 0,80 & 0,51 & Sofrível & 0,30 & 106,55 & 8,98 \\
\hline
\end{tabular}

$\mathrm{R}^{2}$ - coeficiente de determinação da validação cruzada; REMQ - raiz do erro médio quadrático; d - índice de concordância; c - índice de confiança; E' - coeficiente de eficiência ajustado; EMA - erro médio absoluto; e EMP (\%) - erro médio percentual

Analisando os dados da Tabela 3, observa-se que os modelos exponencial e esférico, da técnica da krigagem, obtiveram desempenhos semelhantes e se ajustam melhor aos dados em comparação com o interpolador IPD, com qualquer uma das potências analisadas, porém o modelo esférico se sobressaiu sobre os demais. Os desempenhos dos modelos utilizando a krigagem são considerados bons, exceto o linear, mediante o critério adotado por Camargo \& Sentelhas (1997).

Os dados da Tabela 3 ainda mostram uma pequena variação de EMP entre 7,0 e 8,0\%; em média, o erro percentual do melhor interpolador é muito baixo em se tratando de dados de evapotranspiração potencial, variável que está relacionada com a temperatura que, sabidamente, apresenta grande variação espacial, haja vista que o fator relevo é determinante para isto.

Com o intuito de interpolar valores de ETP a fim de gerar mapas de distribuição espacial deste elemento do clima, Lemos Filho et al. (2007) e Barbosa et al. (2005) utilizaram o método IPD2 para Minas Gerais e Ceará, respectivamente. Em outros trabalhos (Gomes et al., 2005; D’Angiolella et al., 2005) rstudiosos lançaram mão de diferentes interpoladores que não a krigagem, para gerar tais mapas; todavia, não houve qualquer avaliação da eficiência do interpolador nos referidos trabalhos. A Figura 2C e D mostra os mapas contendo a distribuição espacial da ETP no Espírito Santo, obtidos por meio da utilização da krigagem esférica e do IPD2. Da mesma forma que evidenciado para a precipitação, verifica-se tendência no IPD2, de que os valores extremos máximos e mínimos tenham maior influência sobre a interpolação, apresentando maiores áreas nos mapas, quando comparados com o método de krigagem esférica.

Apresentam-se, na Tabela 4, os índices estatísticos calculados para análise do desempenho dos interpoladores para a espacialização da evapotranspiração real média anual, no estado do Espírito Santo.
Tabela 4. Índices estatísticos calculados visando à análise do desempenho dos interpoladores para estimativa da evapotranspiração real média anual, no estado do Espírito Santo

\begin{tabular}{lcrcccccc}
\hline Modelo & $\mathbf{R}^{2}$ & REMQ & $\mathbf{d}$ & $\mathbf{c}$ & Desempenho & $\mathbf{E}^{\prime}$ & EMA & $\begin{array}{c}\text { EMP } \\
\text { (\%) }\end{array}$ \\
Exponencial & 0,59 & 86,04 & 0,86 & 0,66 & Bom & 0,38 & 68,59 & 6,31 \\
Esférico & 0,57 & 89,69 & 0,86 & 0,64 & Mediano & 0,38 & 69,87 & 6,50 \\
Gaussiano & 0,46 & 116,61 & 0,82 & 0,55 & Sofrível & 0,27 & 81,08 & 7,31 \\
Linear & 0,54 & 94,42 & 0,84 & 0,62 & Mediano & 0,37 & 72,41 & 6,64 \\
IPD 1 & 0,42 & 105,58 & 0,75 & 0,49 & Mau & 0,28 & 82,88 & 7,73 \\
IPD 2 & 0,42 & 105,96 & 0,76 & 0,49 & Mau & 0,29 & 81,96 & 7,64 \\
IPD 3 & 0,40 & 108,38 & 0,76 & 0,48 & Mau & 0,28 & 82,91 & 7,72 \\
IPD 4 & 0,37 & 111,67 & 0,76 & 0,47 & Mau & 0,25 & 85,57 & 7,97 \\
IPD 5 & 0,36 & 114,83 & 0,76 & 0,45 & Mau & 0,24 & 87,64 & 8,15 \\
IPD 6 & 0,34 & 117,46 & 0,76 & 0,44 & Mau & 0,22 & 89,45 & 8,32 \\
\hline R2 - coficien
\end{tabular}

$\mathrm{R}^{2}$ - coeficiente de determinação da validação cruzada; REMQ - raiz do erro médio quadrático; $\mathrm{d}$ - índice de concordância; c - índice de confiança; $E^{\prime}$ - coeficiente de eficiência ajustado; EMA - erro médio absoluto; e EMP (\%) - erro médio percentual

O modelo que melhor se ajusta aos dados é o exponencial, uma vez que todos os coeficientes estatísticos apontam nesta direção. Analisando o seu desempenho, constata-se, de acordo com o critério de Camargo \& Sentelhas (1997), que é "bom"; para o IPD, o desempenho é "mau" para todos os níveis de potência.

Poucos trabalhos se propuseram a espacializar a evapotranspiração real no Brasil, se atendo-se apenas à evapotraspiração potencial. D'Angiolella et al. (2005) utilizaram o método de interpolação "spline" para tal fim. A Figura $2 \mathrm{E}$ e $2 \mathrm{~F}$ mostra os mapas contendo a distribuição espacial da ETR no Estado, utilizando-se os métodos krigagem exponencial e spline. Observa-se que o método spline apresentou um mapa completamente diverso daquele obtido por meio da técnica de krigagem indicando, assim, total ineficiência do primeiro quando aplicado exclusivamente no Espírito Santo.

Os resultados da Tabela 5 mostra os índices estatísticos calculados para análise do desempenho dos interpoladores visando a espacialização da deficiência hídrica anual, fator de extrema importância em trabalhos de zoneamento agrícola e que indica o grau de deficiência de água no solo.

Tabela 5. Índices estatísticos calculados visando à análise do desempenho dos interpoladores para estimativa da deficiência hídrica média anual, no estado do Espírito Santo

\begin{tabular}{|c|c|c|c|c|c|c|c|c|}
\hline Modelo & $\mathbf{R}^{2}$ & REMQ & d & c & Desempenho & $\mathbf{E}^{\prime}$ & EMA & $\begin{array}{c}\text { EMP } \\
(\%)\end{array}$ \\
\hline Exponencial & 0,57 & 84,57 & 0,85 & 0,65 & Mediano & 0,43 & 62,87 & 1324,77 \\
\hline Esférico & 0,56 & 85,75 & 0,85 & 0,64 & Mediano & 0,42 & 63,87 & 1344,05 \\
\hline Gaussiano & 0,34 & 118,81 & 0,77 & 0,45 & Sofrível & 0,27 & 78,73 & 1511,80 \\
\hline Linear & 0,56 & 85,54 & 0,86 & 0,64 & Mediano & 0,41 & 64,21 & 1321,50 \\
\hline IPD 1 & 0,48 & 93,25 & 0,80 & 0,55 & Sofrível & 0,32 & 73,59 & 1921,23 \\
\hline IPD 2 & 0,48 & 92,95 & 0,81 & 0,56 & Sofrível & 0,34 & 71,29 & 1936,18 \\
\hline IPD 3 & 0,47 & 94,82 & 0,81 & 0,56 & Sofrível & 0,34 & 71,30 & 1988,52 \\
\hline IPD 4 & 0,45 & 97,72 & 0,81 & 0,54 & Sofrivel & 0,33 & 72,85 & 2078,87 \\
\hline IPD 5 & 0,43 & 100,65 & 0,81 & 0,53 & Sofrível & 0,31 & 74,74 & 2196,39 \\
\hline IPD 6 & 0,42 & 103,23 & 0,80 & 0,52 & Sofrivel & 0,29 & 76,69 & 2326,00 \\
\hline
\end{tabular}

$\mathrm{R}^{2}$ - coeficiente de determinação da validação cruzada; REMQ - raiz do erro médio quadrático; d - índice de concordância; $C$ - índice de confiança; $E^{\prime}$ - coeficiente de eficiência ajustado; EMA - erro médio absoluto; e EMP (\%) - erro médio percentual 
Para esta variável, o modelo de melhor desempenho é a krigagem exponencial, visto que todos os coeficientes estatísticos apontaram nesta direção, quando comparado com os outros métodos; em relação ao seu desempenho, ele é classificado como "mediano", seguindo o critério de Camargo \& Sentelhas (1997).

Ainda de acordo com a Tabela 5, todos os níveis de potência do método IPD obtiveram desempenho considerado "sofrível", indicando que este método não é eficiente para estimar valores deste parâmetro.

O parâmetro deficiência hídrica apresenta altos valores de EMP devido ao grande número de valores reais muito próximos a zero e que levam a altíssimos erros percentuais quando calculados pela Eq. 7. O zero é um limite físico não atendido pelo critério matemático da interpolação. $\mathrm{Na}$ interpolação ocorrem estimativas inferiores a zero, que não existem fisicamente.

$\mathrm{Na}$ Tabela 6 encontrou-se os resultados dos índices estatísticos calculados para análise do desempenho dos interpoladores para a espacialização do excedente hídrico médio anual, no estado do Espírito Santo.

Tabela 6. Índices estatísticos calculados visando à análise do desempenho dos interpoladores para estimativa do excedente hídrico médio anual, no estado do Espírito Santo

\begin{tabular}{lcccccccc}
\hline Modelo & $\mathbf{R}^{2}$ & REMQ & $\mathbf{d}$ & $\mathbf{c}$ & Desempenho & E' & EMA & $\begin{array}{c}\text { EMP } \\
\text { (\%) }\end{array}$ \\
Exponencial & 0,53 & 125,90 & 0,84 & 0,61 & Mediano & 0,42 & 86,98 & 8691,77 \\
Esférico & 0,53 & 126,53 & 0,84 & 0,61 & Mediano & 0,41 & 87,24 & 8041,02 \\
Gaussiano & 0,41 & 156,69 & 0,80 & 0,51 & Sofrível & 0,26 & 111,31 & 10710,62 \\
Linear & 0,53 & 126,49 & 0,84 & 0,61 & Mediano & 0,41 & 87,20 & 8050,55 \\
IPD 1 & 0,50 & 128,87 & 0,82 & 0,58 & Sofrível & 0,34 & 97,54 & 18775,66 \\
IPD 2 & 0,51 & 128,64 & 0,82 & 0,59 & Sofrível & 0,35 & 97,06 & 17311,83 \\
IPD 3 & 0,50 & 131,21 & 0,82 & 0,58 & Sofrível & 0,33 & 99,16 & 16090,67 \\
IPD 4 & 0,48 & 135,03 & 0,82 & 0,57 & Sofrível & 0,32 & 101,32 & 15193,33 \\
IPD 5 & 0,46 & 138,91 & 0,81 & 0,55 & Sofrível & 0,31 & 103,11 & 14552,17 \\
IPD 6 & 0,44 & 142,38 & 0,81 & 0,54 & Sofrível & 0,30 & 104,55 & 14088,12 \\
\hline
\end{tabular}

$\mathrm{R}^{2}$ - coeficiente de determinação da validação cruzada; REMQ - raiz do erro médio quadrático; $\mathrm{d}$ - índice de concordância; $\mathrm{C}$ - índice de confiança; $\mathrm{E}^{\prime}$ - coeficiente de eficiência ajustado; EMA - erro médio absoluto; e EMP (\%) - erro médio percentual.

Os modelos utilizados na krigagem apresentam desempenho semelhante, exceto o gaussiano, porém todos foram superiores aos utilizados no IPD. O modelo exponencial é o que melhor se ajusta, apresentando um desempenho considerado "mediano"; para todos os interpoladores o desempenho é de "sofrível" para "mediano", indicando a dificuldade de ajuste dos dados ao modelo.

A Tabela 7 engloba os índices estatísticos calculados para análise do desempenho dos interpoladores para a espacialização da disponibilidade hídrica média anual, no Espírito Santo.

De acordo com a Tabela 7, o modelo krigagem exponencial possui os melhores índices estatísticos, que o apontam como o melhor método, quando comparado com os outros.

Segundo o critério de Camargo \& Sentelhas (1997), o índice de confiança (c) para o desempenho dos modelos da krigagem é considerado "bom".

Os dados da Tabela 7 mostram, ainda, valores de EMA
Tabela 7. Índices estatísticos calculados visando à análise do desempenho dos interpoladores para estimativa da disponibilidade hídrica média anual, no estado do Espírito Santo

\begin{tabular}{|c|c|c|c|c|c|c|c|c|}
\hline Modelo & $\mathbf{R}^{2}$ & REMQ & d & c & Desempenho & $\mathbf{E}^{\prime}$ & EMA & $\begin{array}{c}\text { EMP } \\
(\%)\end{array}$ \\
\hline Exponencial & 0,60 & 0,06 & 0,87 & 0,67 & Bom & 0,42 & 0,05 & 5,38 \\
\hline Esférico & 0,59 & 0,06 & 0,86 & 0,66 & Bom & 0,41 & 0,05 & 5,53 \\
\hline Gaussiano & 0,38 & 0,08 & 0,79 & 0,49 & Mau & 0,27 & 0,06 & 6,7 \\
\hline Linear & 0,59 & 0,06 & 0,87 & 0,66 & Bom & 0,41 & 0,05 & 5,54 \\
\hline IPD 1 & 0,51 & 0,07 & 0,81 & 0,58 & Sofrível & 0,34 & 0,05 & 6,22 \\
\hline IPD 2 & 0,51 & 0,07 & 0,82 & 0,59 & Sofrível & 0,36 & 0,05 & 6,09 \\
\hline IPD 3 & 0,50 & 0,07 & 0,82 & 0,58 & Sofrível & 0,36 & 0,05 & 6,11 \\
\hline IPD 4 & 0,48 & 0,07 & 0,82 & 0,57 & Sofrível & 0,34 & 0,05 & 6,25 \\
\hline IPD 5 & 0,46 & 0,07 & 0,82 & 0,55 & Sofrível & 0,32 & 0,05 & 6,41 \\
\hline IPD 6 & 0,45 & 0,07 & 0,81 & 0,54 & Sofrível & 0,31 & 0,05 & 6,58 \\
\hline
\end{tabular}

$\mathrm{R}^{2}$ - coeficiente de determinação da validação cruzada; REMQ - raiz do erro médio quadrático; $\mathrm{d}$ - índice de concordância; $\mathrm{C}$ - índice de confiança; $E^{\prime}$ - coeficiente de eficiência ajustado; EMA - erro médio absoluto; e EMP (\%) - erro médio percentual

que variam entre 0,5 e 0,6 ; fato que se justifica, pois os valores de disponibilidade hídrica (ER/ETP) variam entre 0 e 1 , sendo que, quanto mais próximo de 1 , menor o risco climático de não atendimento hídrico.

Sentelhas et al. (2008) utilizaram o modelo spline para interpolar os parâmetros deficiência hídrica e excedente hídrico no Brasil, todavia sem qualquer avaliação quanto à sua eficiência; o mesmo ocorreu com o trabalho de D'Angiolella et al. (2005) para os parâmetros EXC, DEF e ER/ETP para o sul da Bahia. Cecílio et al. (2006) e Silva et al. (2007) observaram que o modelo Spline apresentou os piores resultados em relação aos métodos de Krigagem e IPD2, com melhores resultados para o segundo, para a interpolação dos parâmetros déficit hídrico e disponibilidade hídrica e do primeiro para o parâmetro excedente hídrico; já Macedo et al. (2001) apontaram para a importância da krigagem na interpolação da disponibilidade hídrica, chamada pelos autores Índice de satisfação das necessidades de água. Para fins de comparação, parece haver certa tendência na utilização da spline.

A Figura 3A, B, C, D, E e F mostra, para o Espírito Santo, os mapas contendo a distribuição espacial do DEF, EXC e ER/ETP, respectivamente. Foram utilizados os métodos krigagem exponencial (Figura 3A, C e E) e spline (Figura $3 \mathrm{~B}, \mathrm{D}$ e $\mathrm{F})$.

Observaram-se, conforme resultado também evidenciado para a variável ETR, grandes diferenças entre os mapas obtidos por krigagem e por spline. O método spline chegou a estimar valores negativos para os parâmetros DEF e EXC, o que foge à realidade física indicando, mas uma vez, sua ineficiência para a área em estudos.

Os resultados obtidos vêm constatar a superioridade do interpolador krigagem para estimativa dos parâmetros do balanço hídrico no Espírito Santo, o que é atribuído, segundo Vieira (2000), devido ao fato de o método estar embasado na não-tendenciosidade do estimador e variância mínima das estimativas. Desta forma, corroborando Carvalho \& Assad (2005), os valores interpolados por meio da krigagem são não-viciados, têm variância mínima e são ideais para a construção de mapas de isolinhas ou 
A.

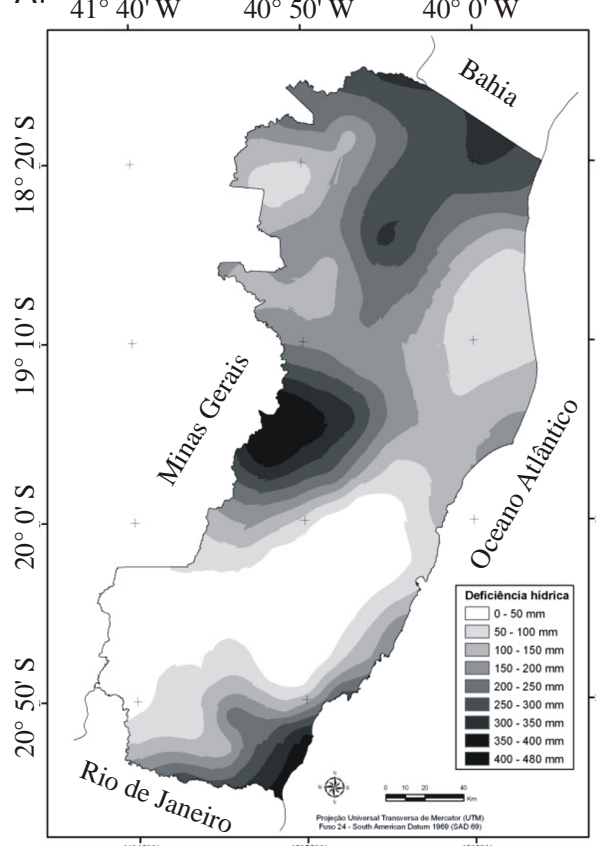

D. $41^{\circ} 40^{\prime} \mathrm{W} \quad 40^{\circ} 50^{\prime} \mathrm{W} \quad 40^{\circ} 0^{\prime} \mathrm{W}$

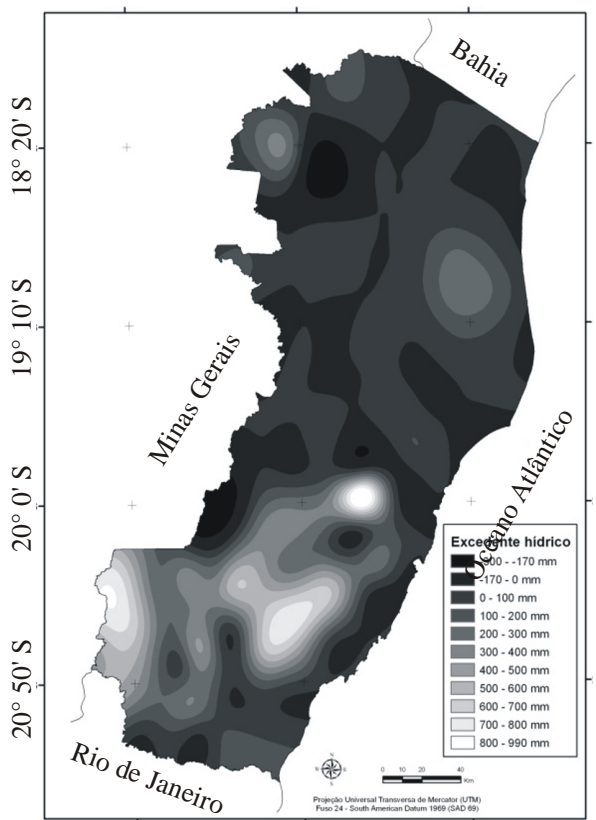

$41^{\circ} 40^{\prime} \mathrm{W} \quad 40^{\circ} 50^{\prime} \mathrm{W} \quad 40^{\circ} 0^{\prime} \mathrm{W}$
B.

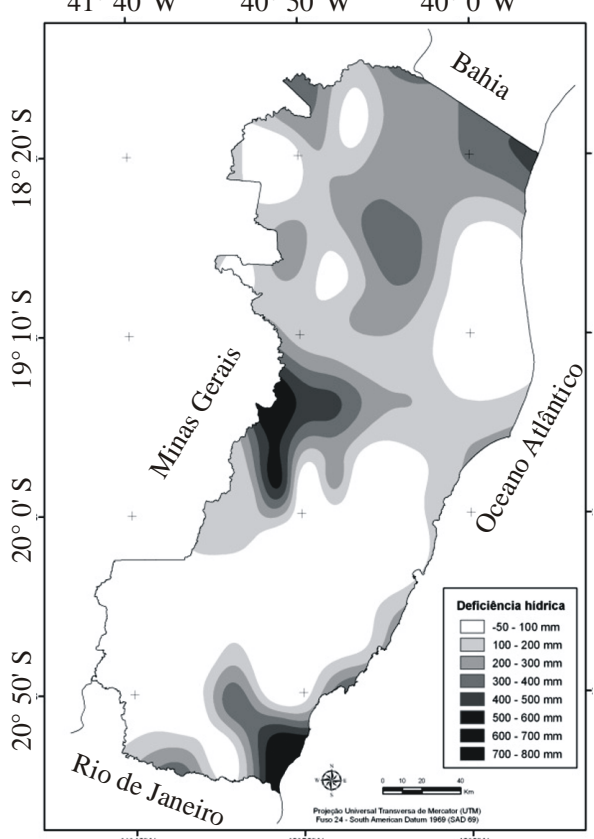

C.

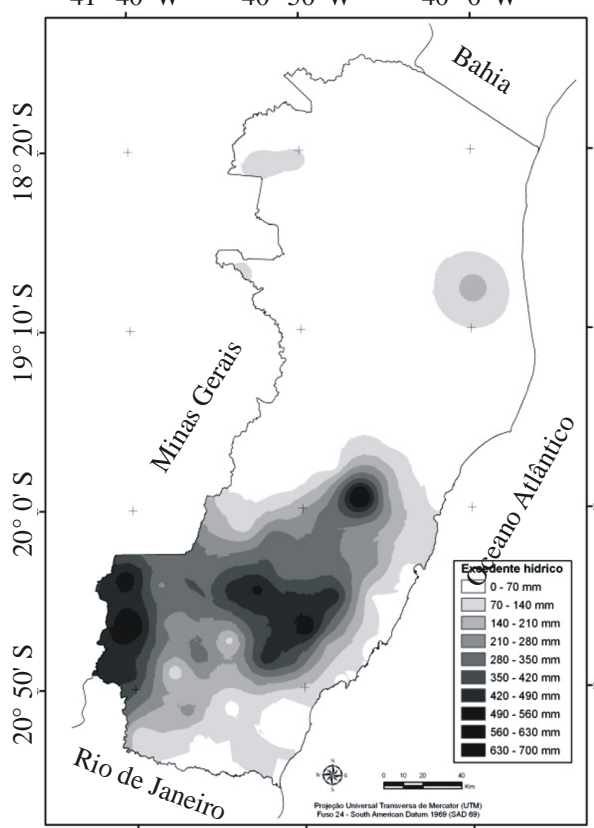

E. $41^{\circ} 40^{\prime} \mathrm{W} \quad 40^{\circ} 50^{\prime} \mathrm{W} \quad 40^{\circ} 0^{\prime} \mathrm{W}$

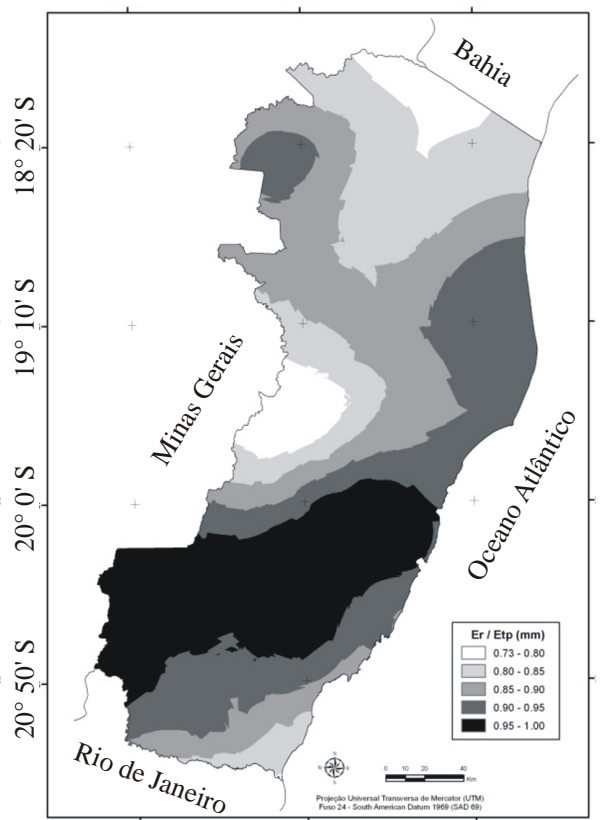

$41^{\circ} 40^{\prime} \mathrm{W} \quad 40^{\circ} 50^{\prime} \mathrm{W} \quad 40^{\circ} 0^{\prime} \mathrm{W}$
F. $41^{\circ} 40^{\prime} \mathrm{W} \quad 40^{\circ} 50^{\prime} \mathrm{W} \quad 40^{\circ} 0^{\prime} \mathrm{W}$

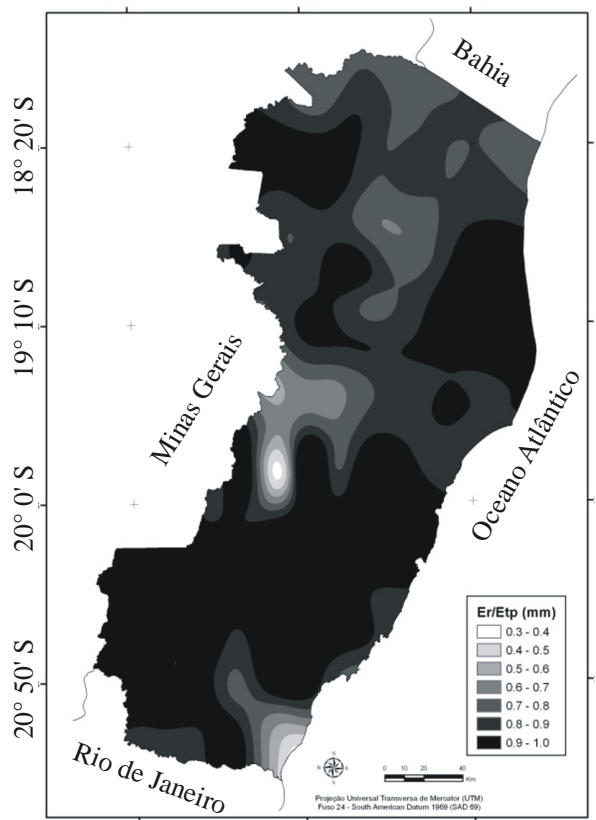

$41^{\circ} 40^{\prime} \mathrm{W} \quad 40^{\circ} 50^{\prime} \mathrm{W} \quad 40^{\circ} 0^{\prime} \mathrm{W}$

Figura 3. Distribuições espaciais do déficit hídrico anual no Espírito Santo, obtidas por meio de krigagem exponencial (A) e spline (B); Distribuicões espaciais do excedente hídrico anual no Espírito Santo, obtidas por meio de krigagem exponencial (C) e spline (D); distribuições espaciais da disponibilidade hídrica anual no Espírito Santo, obtidas por meio de krigagem exponencial (E) e spline (F)

tridimensionais para a verificação e a interpretação da variabilidade espacial de elementos climáticos sendo, desta forma, muito úteis para se entender a variabilidade das características climáticas de uma região e identificar áreas com maior ou menor favorabilidade de desenvolvimento de culturas e ocorrência de patógenos.

Convém ressaltar que os resultados aqui apresentados dizem respeito aos interpoladores de melhor desempenho para a espacialização dos elementos climáticos estudados apenas para a área do estado do Espírito Santo. A utilização desses interpoladores em diferentes regiões devem ser feita com critério, avaliando previamente seu desempenho; além disso, é oportuno ressaltar que se avaliaram os parâmetros DEF, EXC e ER/ETP, obtidos por meio de balanço hídrico considerando um valor de CAD igual a $100 \mathrm{~mm}$. A utilização de diferentes valores para CAD altera os resultados do balanço hídrico, o que pode provocar mudanças no desempenho dos interpoladores; contudo, são necessários estudos que confirmem a magnitude da alteração do desempenho. 


\section{CONCLUSÕES}

1. A escolha do modelo de interpolação que apresentou o melhor desempenho foi de extrema importância para a realização do processo de interpolação das variáveis estudadas.

2. A Krigagem é o método de interpolação que apresenta as melhores estimativas das variáveis precipitação, evapotranspiração potencial, evapotranspiração real, deficiência hídrica, excedente hídrico e disponibilidade hídrica.

\section{LITERATURA CITADA}

Amorim, R. C. F.; Ribeiro, A.; Leite, C. C.; Leal, B. G.; Silva, J. B. G. Avaliação do desempenho de dos métodos de espacialização da precipitação pluvial para o estado de Alagoas. Acta Scientiarum Technology, v.30, n.1, p.87-91, 2008.

Aranoff, S. Geographic information systems: a management perspective. Ottawa: WDL Publications, 1989. 294p.

Barbosa, F. C.; Teixeira, A. S.; Gondim, R. S. Espacialização da evapotranspiração de referência e precipitação efetiva para estimativa das necessidades de irrigação na região do Baixo Jaguaribe-CE. Revista Ciência Agronômica, v.36, n.1, p.24-33, 2005.

Barbosa, J. P. M. Utilização de método de interpolação para análise e espacialização de dados climáticos: O Sig como ferramenta. Caminhos da Geografia (UFU), v.9, n.17, p.85-96, 2006.

Bertoni, J. C.; Tucci, C. E. M. Precipitação In: Tucci, C. E. M. (org.). Hidrologia: Ciência e aplicação. 3.ed. Porto Alegre: UFRGS/ABRH, 2002. p.177-231.

Black, P. Revising the Thornthwaite and Mather water balance. Journal of the American Water Resources Association, v.43, n.6, p.1604-1605, 2007.

Camargo, A. P.; Sentelhas, P. C. Avaliação do desempenho de diferentes métodos de estimativa da evapotranspiração potencial no estado de São Paulo, Brasil. Revista Brasileira de Agrometeorologia, v.5, n.1, p.89-97, 1997.

Carvalho, J. R. P.; Assad, E. D. Análise espacial da precipitação pluviométrica no estado de São Paulo: Comparação de métodos de interpolação. Engenharia Agrícola, v.25, n.2, p.377-384, 2005.

Cecílio, R. A.; Medeiros, S. S.; Dantas Neto, F. S. Zoneamento edafoclimático da Sub-Bacia do Rio Paranaíba, em Minas Gerais para o cultivo dos cafeeiros arábica (Coffea arabica L.) e conilon (Coffea canephora L.). Caminhos da Geografia (UFU), v.6, n.19, p.113-125, 2006.

Chong, S. K.; Green, R. E.; Ahuja, L. R. Infiltration prediction based on estimation of Green-Ampt wetting front pressure head from measurements of soil water redistribution. Soil Science Society of America Journal, v.46, p.235-239, 1982.

D’Angiolella, G.; Vasconcellos, V. L. D.; Rosa, J. W. C. Estimativa e espacialização do balanço hídrico na mesorregião sul da Bahia. In: Simpósio Brasileiro de Sensoriamento Remoto, 12, 2005, Goiânia. Anais... Goiânia: INPE, 2005. p.83-90.
Gomes, A. A. N.; Andrade Júnior, A. S.; Medeiros, R. M. Evapotranspiração de referência mensal para o Estado do Piauí. Revista Brasileira de Engenharia Agrícola e Ambiental, v.9, n.4, p.560-564, 2005.

Legates, D. R.; McCabe Jr., G. J. Evaluating the use of "goodness-of-fit" measures in hydrologic and hydroclimatic model validation. Water Resources Research, v.35, n.1, p.233-241, 1999.

Lemos Filho, L. C. A.; Carvalho, L. G.; Evangelista, A. W. P.; Carvalho, L. M. T.; Dantas, A. A. A. Análise espaço-temporal da evapotranspiração de referência para Minas Gerais. Ciência \& Agrotecnologia, v.31, n.5, p.1462-1469, 2007.

Lennon, J. J.; Turner, J. R. G. Predicting the spatial distribution of climate: temperature in Great Britain. Journal of Animal Ecology, n.64, p.392-670, 1995.

Macedo, M. A.; Assad, E. D.; Câmara, G.; Oliveira, J. C.; Barbosa, A. M. Avaliação de métodos para espacialização de índices de necessidade hídrica das culturas e sua aplicação em zoneamento agrícola. Revista Brasileira de Agrometeorologia, v.9, n.3, p.581-587, 2001.

Mathworks. Software MATLAB 2007b, MathWorks, 2007.

Nalder, I. A.; Wein, R. W. Spatial interpolation of climatic Normals: test of a new method in the Canadian boreal Forest. Agricultural and Forest Meteorology, v. 92, p.211-225, 1998.

Pereira, A. R.; Angelocci, L. R.; Sentelhas, P. C. Agrometeorologia: fundamentos e aplicações práticas. Guaíba: Agropecuária, 2002. 478p.

Price, D. T.; McKenney, D. W.; Nalder, I. A.; Hutchinson, M. F.; Kesteven, J. L. A comparison of two statistical methods for spatial interpolation of Canadian monthly mean climate data. Agricultural and Forest Meteorology, v.101, p.81-94, 2000.

Robinson, T. P.; Metternicht, G. Testing the performance of spatial interpolation techniques for mapping soil properties. Computers and Electronics in Agriculture, v.50, n.2, p. $97-108,2006$.

Rolim, G. S.; Sentelhas, P. C.; Barbieri, V. Planilhas no ambiente EXCEL para os cálculos de balanços hídricos: normal, sequencial, de cultura e de produtividade real e potencial. Revista Brasileira de Agrometeorologia, v.6, p.133-137, 1998.

Sentelhas, P. C.; Santos, D. L.; Machado, R. E. Water deficit and water surplus maps for Brazil, based on FAO Penman-Monteith potential evapotranspiration. Ambiágua, v.3, n.3, p.28-42, 2008.

Silva, K. R.; Paiva, Y. G.; Cecílio, R. A.; Pezzopane, J. E. M. Avaliação de interpoladores para a espacialização de variáveis climáticas na bacia do rio Itapemirim-ES. In: Simpósio Brasileiro de Sensoriamento Remoto, 13, 2007, Florianópolis. Anais. São José dos Campos: INPE, 2007. p.3141-3146.

Souza, L. S.; Cogo, N. P.; Vieira, S. R. Variabilidade de fósforo, potássio e matéria orgânica em relação a sistemas de manejo. Revista Brasileira de Ciência do Solo, v. 22, p.77-86, 1998.

Taylor, S. P.; Haywood, A. M.; Valdes, P. J.; Sellwood, B. W. An evaluation of two spatial interpolation techniques in global seasurface temperature reconstructions: Last glacial maximum and pliocene case studies. Quaternary Science Reviews, v.23, p.1041-1051, 2004. 
Thornthwaite, C. W. An approach toward a rational classification of climate. Geografical Review, v.38, p.55-94, 1948.

Thornthwaite, C. W.; Mather, J. R. The water balance. Publications in Climatology. New Jersey: Drexel Institute of Technology, 1955. 104p.

Vieira, S. R. Variabilidade espacial de argila, silte e atributos químicos de uma parcela experimental de um Latossolo roxo de Campinas - SP. Bragantia, v.1, n.56, p.181-190, 1997.

Vieira, S. R. Geoestatística em estudos de variabilidade espacial do solo. In: Novais, R. F.; Alvarez V., V. H.; Schaefer, C. E. G. R. (ed.) Tópicos em ciência do solo. Viçosa: SBCS, p.1-54. 2000.
Wilcox, B. P.; Rawls, W. J.; Brakensiek, D. L.; Wight, J. R. Predicting runoff from rangeland catchments: a comparison of two models. Water Resources Research, v.26, p.2401-2410, 1990.

Xavier, A. C.; Cecílio, R. A.; Lima, J. S. S. Módulos em Matlab para interpolação espacial pelo método de krigagem ordinária e do inverso da distância. Revista Brasileira de Cartografia, v.62, n.1, p.67-76, 2010.

$\mathrm{Yu}, \mathrm{B}$. A comparison of the Green-Ampt and a spatially variable infiltration model for natural storm events. Transactions of the ASAE, v.42, n.1, p.88-97, 1999. 